\title{
Свойства податливых подложек на основе пористого кремния, сформированных двухстадийным травлением
}

\author{
(c) П.В. Середин ${ }^{1}$, А.С. Леньшин ${ }^{1}$, Abduljabbar Riyad Khuder ${ }^{2}$, Д.Л. Голощапов ${ }^{1}$, \\ М.А. Хараджиди ${ }^{1}$, И.Н. Арсентьев ${ }^{3}$, И.А. Касаткин ${ }^{4}$ \\ ${ }^{1}$ Воронежский государственный университет, \\ 394018 Воронеж, Россия \\ ${ }^{2}$ Ministry of Education/General Directorate of Education in Baghdad, \\ The third Karkh Governorate, Iraq \\ ${ }^{3}$ Физико-технический институт им. А.Ф. Иоффе Российской академии наук, \\ 194021 Санкт-Петербург, Россия \\ ${ }^{4}$ Санкт-Петербургский государственный университет, \\ 199034 Санкт-Петербург, Россия \\ E-mail: paul@phys.vsu.ru
}

Поступила в Редакцию 5 июля 2021 г.

В окончательной редакции 12 июля 2021 г.

Принята к публикации 12 июля 2021 г.

\begin{abstract}
Сообщается о влиянии режимов травления и их комбинации на дизайн, микроструктурные и оптические свойства податливых подложек на основе пористого кремния. На основе данных комплекса микроструктурных и спектроскопических методов анализа показано, что при неизменных параметрах кристаллической решетки величина остаточных напряжений, размер кристаллитов, объем кристаллической фракции, а также отражательная способность и энергия прямых переходов в пористом слое кремния зависят от комбинации режимов травления, однако не всегда коррелируют с величиной пористости слоя, рассчитанной из анализа SEM изображений.
\end{abstract}

Ключевые слова: $\mathrm{Si}$, пористый слой, податливая подложка.

DOI: 10.21883/FTP.2021.11.51555.9707

\section{1. Введение}

Кремний является не только главным материалом современной электроники, но и в перспективе фотоники будущего [1]. Интегрированные источники света, совмещающие в едином устройстве преимущества кремниевой технологии и уникальные оптические и электрофизические свойства передовых полупроводниковых материалов группы GaAs и нитридов $\mathrm{A}^{\mathrm{III}} \mathrm{N}$, являются важным компонентом в широком спектре приложений фотоники [2]. Одним из методов интеграции $\mathrm{A}^{\mathrm{III}} \mathrm{B}^{\mathrm{V}} \mathrm{c}$ $\mathrm{Si}$ является рост на виртуальных подложках на основе нанопористого кремния [3], либо нанопрофилированной поверхности кремния с существенно развитым рельефом (протопористый кремний). Использование данного подхода положительно отражалось на оптических и структурных свойствах гибридных $\mathrm{A}^{\mathrm{III}} \mathrm{B}^{\mathrm{V}} / \mathrm{Si}$ или $\mathrm{A}^{\mathrm{III}} \mathrm{N} / \mathrm{Si}$ гетероструктур [4-7].

Говоря о пористом кремнии, полученном методом электрохимического травления, важно отметить, что сам по себе он уже является перспективным и востребованным материалом для производства фотонных структур [8], солнечных элементов, волноводов [9]. И хотя за последнее время был достигнут значительный прогресс в продвижении пористых слоев кремния [10,11], полученных методом электрохимического травления, в том числе как податливой подложки при создании высокоэффективных устройств гибридной $\mathrm{A}^{\mathrm{III}} \mathrm{B}^{\mathrm{V}} / \mathrm{Si}$ и $\mathrm{A}^{\mathrm{III}} \mathrm{N} / \mathrm{Si}$ электроники, масштабируемый и управляемый синтез наноструктурных пористых слоев $\mathrm{Si}$ с заданными стабильными свойствами остается проблемой.

Так, одной из остро стоящих задач является контроль глубины, шероховатости и, что немаловажно, структурных характеристик податливой пористой подложки.

На наш взгляд, одним из вариантов решения указанной проблемы является использование податливой пористой подложки, имеющей в своем составе несколько пористых слоев с изменяемой пористостью в каждом подслое. Такие структуры можно эффективно получить за счет чередования разных импульсов тока [8]. В результате появляется возможность эффективно перераспределять напряжения кристаллической решетки по глубине пористого слоя податливой подложки. Основной задачей, которую предстоит здесь решить, является исследование влияния режимов стадийного травления и их комбинации на дизайн, структурные и оптические свойства податливых подложек на основе пористого кремния. Этой проблеме посвящена наша работа.

\section{2. Материалы и методы исследования}

Слои пористого кремния были сформированы методом электрохимического травления по отработанной нами методике $[12,13]$ в растворе плавиковой кислоты и диметилформамида с добавлением перекиси водорода и серной кислоты. При этом использовались подложки (wafers) кремния, легированные фосфором 

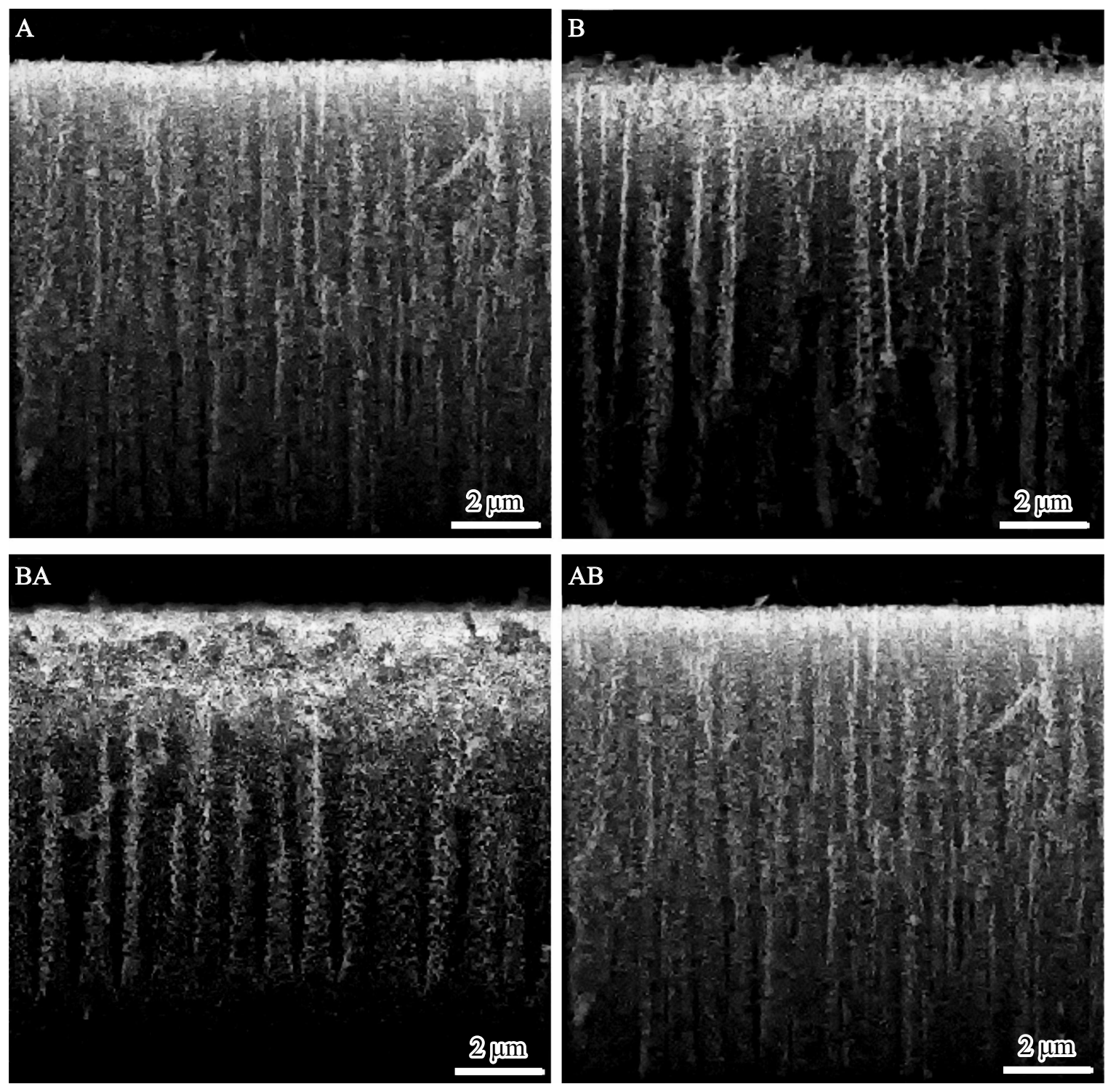

Рис. 1. СЭМ-изображения сколов образов пористого кремния, полученного разностадийным травлением (см. табл. 1).

(0.3 Ом · см, n-). Были подобраны режимы одностадийного или двухстадийного электрохимического травления (см. табл. 1). Режимы двухстадийного травления являлись последовательной комбинацией одностадийных режимов.

Полученные образцы были изучены комплексом структурно-спектроскопических и микроскопических методов анализа.

Таблица 1. Режимы получения образцов пористого кремния

\begin{tabular}{c|c|c|c}
\hline № & Режим травления & $\begin{array}{c}\text { Плотность анодного } \\
\text { тока, мА/см² }\end{array}$ & $\begin{array}{c}\text { Время, } \\
\text { мин }\end{array}$ \\
\hline $\mathrm{A}$ & Одностадийный & 15 & 10 \\
$\mathrm{~B}$ & То же & 50 & 10 \\
$\mathrm{BA}$ & Двухстадийный & $50 / 15$ & $5 / 5$ \\
$\mathrm{AB}$ & То же & $15 / 50$ & $5 / 5$
\end{tabular}

Рентгеновские кривые качания были получены при $305 \mathrm{~K}$ с помощью дифрактометра Bruker D8 Discover, работающего в режиме линейного фокуса с параллельным пучком. Первичный пучок монохроматизирован для получения излучения $\mathrm{Cu} K_{\alpha 1}$ с длиной волны $1.54056 \AA$.

Поперечные исследования слоев пористого кремния выполнены с использованием сканирующего электронного микроскопа (СЭМ) JEOL JSM 6380 LV.

Спектры комбинационного рассеяния (рамановские спектры) были получены на конфокальном рамановском микроскопе RamMix 532 со спектральным разрешением $2 \mathrm{~cm}^{-1}$ в диапазоне $200-2000 \mathrm{~cm}^{-1}$. Возбуждение проводилось лазером с длиной волны 532 нм, мощность на образце $\sim 10-50$ мВт. Сигнал с поверхности образца собирали с использованием объектива 100х. Предварительно спектрометр был тщательно откалиброван с использованием эталонного спектра полистирола. Для 
увеличения отношения сигнал/шум полезный сигнал был усреднен на основе 100 сканирований.

\section{3. Результаты исследования и обсуждение}

Тип и последовательность режимов травления пластин кремния влияют на структуру формируемого пористого слоя. На рис. 1 изображены поперечные сечения сколов четырех типов пористого кремния, полученных одно- или двухстадийным (комбинированным) травлением.

Эксперимент показывает, что подобранные режимы травления обеспечили формирование пористого слоя толщиной не менее 10-15 мкм для всех образцов. При этом СЭМ-изображения демонстрируют образование перпендикулярных поверхности образца колонн, некоторые из которых имеют множество ярко выраженных ответвлений (образцы А, В). Кроме того, как видно из СЭМ данных, для образцов А, В, ВА характерно наличие более растравленного поверхностного подслоя, максимальная толщина которого $\sim 2$ мкм характерна для образца ВА. Диаметр пор в образцах варьируется от режима травления и составляет в среднем для образцов: $\mathrm{A}-\sim 270, \mathrm{~B}-\sim 350, \mathrm{BA}-\sim 410, \mathrm{AB}-$ $\sim 240$ нм. Расстояние между колоннами также зависит от последовательности стадий травления и составляет для образцов: А $\sim 210, \mathrm{~B}-\sim 140$, ВА $-\sim 370$, $\mathrm{AB} \sim \sim 140 \mathrm{HM}$.

Исследование пористости сформированных слоев осуществлялось на основе обработки СЭМ-изображений с использованием алгоритмов, описанных в работах $[14,15]$. Как следует из СЭМ-изображений сколов, поры в слоях имеют приближенно цилиндрическую форму, что является допущением в наших расчетах при вычислении среднего диаметра пор, а также размера стенки, которая разделяет поры. Пористость слоя определялась как отношение общего объема пор к объему всего слоя. Полагая, что поры распределены по всей толщине однородно, отношение между полными областями темных элементов на СЭМ-изображении и всей сканированной областью должно представлять собой соотношение, отражающее пористость образца $[14,15]$. Выполнив профилирование поверхности и вычислив площадь, приходящуюся на пористую часть образца, и площадь всей поверхности с учетом геометрии пор,

Таблица 2. Результаты анализа СЭМ-изображений и данные рентгенодифракционного анализа

\begin{tabular}{c|c|c|c}
\hline № & $P, \%$ & Мозаичность, $\eta$ & $\sigma$, ГПА \\
\hline A & 58 & 0.229 & 0.700 \\
В & 69 & 0.325 & 0.800 \\
BA & 54 & 0.195 & 0.525 \\
AB & 60 & 0.239 & 0.750
\end{tabular}

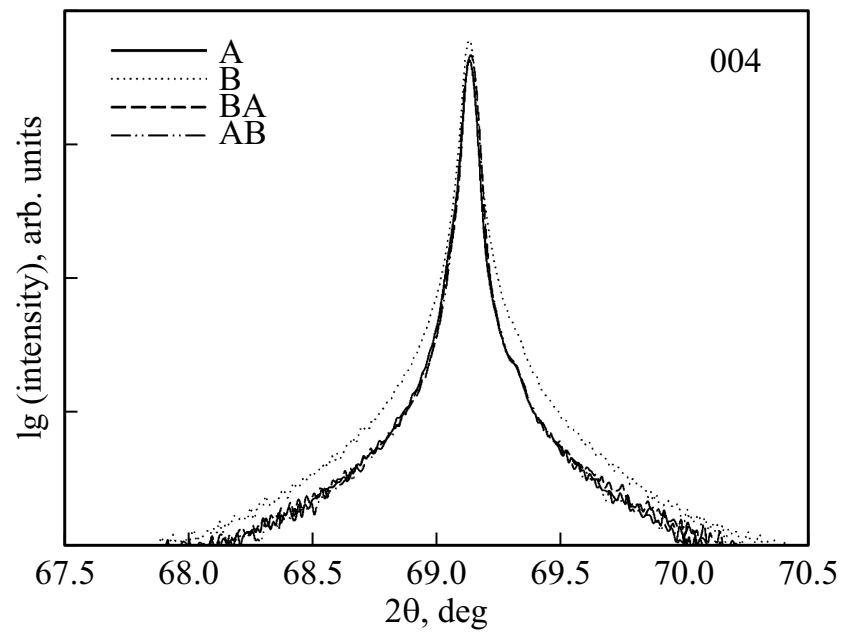

Рис. 2. Q-2Q сканы образцов пористого кремния.

нами были получены средние значения пористости, представленные в табл. 2.

Влияние режимов формирования пористого слоя на структурные характеристики образцов мы оценили на основе высокоразрешающей дифракции рентгеновских лучей.

На рис. 2 представлены Q-2Q сканы образцов в области 004 рефлекса кремния.

Хорошо видно, что на дифрактограммах присутствует только один рефлекс, совпадающий по положению с отражением от монокристаллической подложки, и не наблюдается дополнительных максимумов от слоя пористого кремния. Этот факт совпадает с данными работы [9], в которой исследовали микроструктуру и кристалличность однослойных образцов пористого кремния, созданных на слабо легированных фосфором подложках 1-3 Ом·см, аналогичных тем, что использовались в нашей работе. Также хорошо видно, что ширины Q-2Q пиков практически идентичны, за исключением образца $\mathrm{B}$, чья полуширина незначительно больше. Это указывает на тот факт, что с изменением режимов травления размер кристаллитов у образцов является величиной одного порядка.

Для определения величины кристалличности нами были получены симметричные 004 кривые качания с высоким разрешением. Данные представлены на рис. 3. Здесь также представлена кривая качания эталонного образца монокристаллического кремния. Как видно из представленных данных, на кривых качания всех образцов также можно наблюдать только пик кремниевой подложки. При этом под рефлексом присутствует характерный четкий горб, что также указывает на перекрытие отражения от пористого слоя с отражением подложки. Различное положение 004 максимума на кривых качания относительно положения эталона $\mathrm{Si}$ является следствием наличия разориентации у использованной подложки $\mathrm{Si}$ относительно направления $\langle 001\rangle$. Анализ кривых качания показал (см. рис. 3), что для эталонного образца 


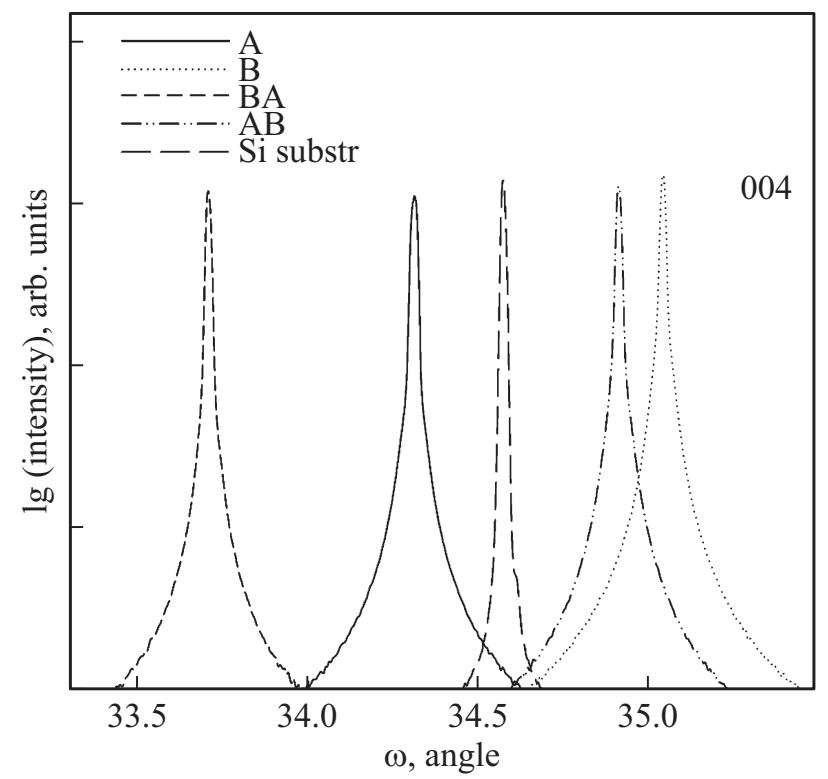

Рис. 3. Рентгеновские кривые качания 004 (слева) образцов пористого кремния, полученного разностадийным травлением.

ширина кривой совпадает с инструментальной шириной в аналогичных условиях. При этом ширины рефлексов 004 на кривых качания пористых слоев заметно больше, что является следствием формирования пористого слоя. Травление приводит к возникновению мозаичности, т.е. кристаллиты в пористом слое слегка разориентируются по отношению друг к другу. Поскольку кривая качания является функцией мозаичности кристаллов, ее угловая ширина $\Delta \omega_{R C}$ будет определяться углом разворота блоков относительно друг друга, т.е. мозаичностью $\eta$. В итоге угловой диапазон разориентации кристаллитов в пористом слое может быть оценен как разница ширины кривой качания и инструментальной ширины. Эти данные представлены в табл. 2.

Анализ рассчитанной величины разориентации мозаичных блоков в пористом слое показывает, что увеличение величины пористости, определенной из анализа СЭМ-изображений и достигаемой в том числе комбинацией режимов травления, коррелирует с мозаичностью.

Использование рамановской микроспектроскопии позволило нам исследовать тонкие структурные свойства, состав и качество кристаллов в образцах на основе изучения изменений, происходящих в колебательном фононном спектре решетки, вызванных процессами образования пористой структуры. Измерение рамановского рассеяния проводилось в геометрии с падающим и рассеянным светом, направленным вдоль оси [001]. В соответствии с теорией в рамановских спектрах будут наблюдаться продольные оптические фононы (LO), рассеянные с поверхности (001) кремния.

Профили типичного рамановского рассеяния для сформированных нами образцов пористого кремния, а также спектр монокристаллической подложки $\mathrm{Si}$ в области $460-560 \mathrm{~cm}^{-1}$ представлены на рис. 4.
Хорошо видно, что рамановский спектр подложки в области 460-560 $\mathrm{cm}^{-1}$ содержит один высокоинтенсивный симметричный максимум около $\sim 521 \mathrm{~cm}^{-1}$, соотносимый c LO фононной модой в центральной точке зоны Бриллюэна и характеризуемый малой шириной на половине высоты $(\mathrm{FWHM}) \sim 5.5 \mathrm{~cm}^{-1}$. Что касается профилей рамановского рассеяния слоев пористого кремния, то, как видно из рис. 4 , они представляют собой асимметричные полосы, с широкой хвостовой частью, при этом главный максимум полос сдвинут в низкочастотную область спектра относительно положения для монокристаллической подложки.

Неоднократно было показано, что использование микрорамановской спектрокопии является эффективным экспериментальным методом исследования остаточных напряжений в структурах с слоями пористого кремния [16]. Сдвиг LO-моды кремния в протравленных слоях кремния с разной степенью пористости относительно позиции LO-моды для монокристаллической подложки позволяет оценить остаточные напряжения [17] в соответствии со следующим соотношением:

$$
\Delta \omega=-4 \cdot 10^{-10} \sigma .
$$

Здесь $\Delta \omega-$ частотный сдвиг, а величина остаточных

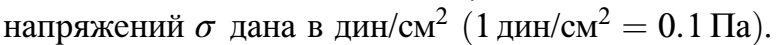

Расчет показывает (см. табл. 1), что величина остаточных напряжений лежит в пределах 525-800 МПа, что согласуется с данными работы [18] для однослойных структур с аналогичными величинами пористости. Однако необходимо отметить, что в соответствии с работой [16] остаточные напряжения достаточно сложно распределяются внутри всей пористой кремниевой структуры. Поскольку глубина анализа с использованием рамановской спектроскопии составляет 600-700 нм, то точное определение напряжений, их распределения и

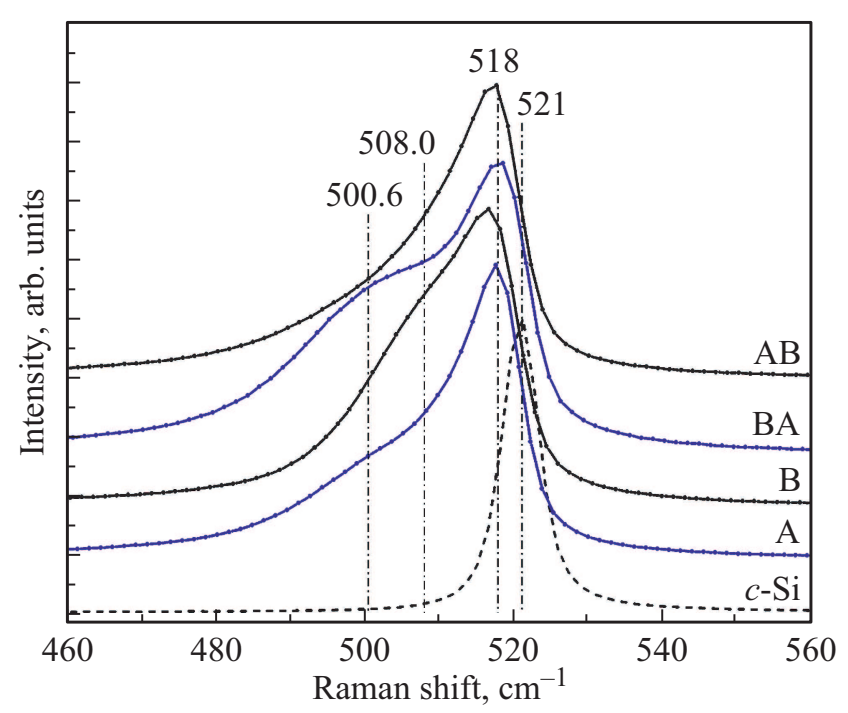

Рис. 4. Рамановские спектры слоев пористого кремния и монокристаллической подложки $\mathrm{Si}$ в области $460-560 \mathrm{~cm}^{-1}$. 


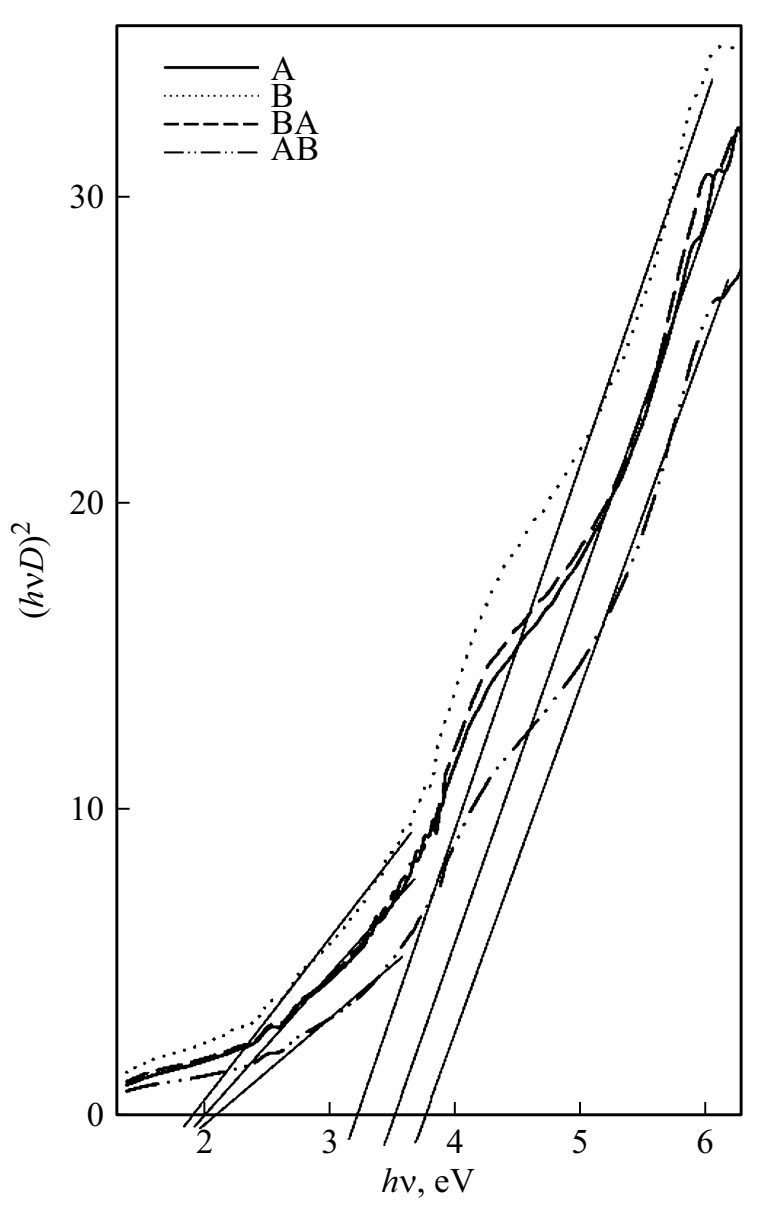

Рис. 5. Зависимости $(D \cdot h v)^{2}$ от энергии квантов для образцов пористого кремния. (Цветной вариант рисунка представлен в электронной версии статьи).

градиента требует проведения локального микрокартирования скола пористого слоя, что является отдельной задачей.

Оптические свойства в UV-диапазоне пористых слоев кремния, полученных разностадийным травлением, были изучены с использованием хорошо зарекомендовавшей себя методики съемки на отражение $[6,19]$, позволяющей получать информацию от тонких слоев, сформированных на оптически более плотных подложках. Для выявления механизмов оптического поглощения и оптических переходов, характерных для пористых образцов, спектры пропускания-отражения были перестроены в зависимости $(D \cdot h v)^{2}$ от энергии квантов (рис. 5). Для этого мы использовали возможности программы Omnic. Здесь $D-$ оптическая плотность. Дальнейший графический анализ этих зависимостей позволил нам обнаружить линейные участки. Линейная экстраполяция данных участков к нулевому значению позволила определить два вида прямых разрешенных переходов для образцов (рис. 5).

Тщательный анализ показывает, что высокоэнергетические прямые переходы в сформированных слоях пористого кремния лежат в области $3.2-3.7$ эВ. В со- ответствии с литературными данными - это переходы из валентной зоны в зону проводимости и $\Lambda_{2} \rightarrow \Lambda_{1}$ [20]. Низкоэнергетические переходы расположены в области 1.85-2.05 эВ и могут быть приписаны эмиссии от пористого кремния с характерным размером пор [12]. При этом стоит отметить, что сдвиг по энергии как высоко, так и низкоэнергетических переходов зависит от типа и последовательности примененного травления и имеет обратную корреляцию с величиной коэффициента отражения.

\section{4. Заключение}

Использование режимов одностадийного травления и их комбинаций позволило нам сформировать пористые слои кремния приблизительно одной толщины, но при этом обладающие различными структурными, морфологическими и оптическими свойствами. На основе данных комплекса структурно-спектроскопических и микроскопических методов анализа удалось показать, что при неизменных параметрах кристаллической решетки величина остаточных напряжений, размер кристаллитов, а также отражательная способность и энергия прямых переходов в пористом слое кремния зависят от комбинации режимов травления, однако не всегда коррелируют с величиной пористости слоя, рассчитанной из анализа СЭМ-изображений. Грамотный подбор указанных параметров может обеспечить дополнительные преимущества при дальнейшей монолитной интеграции $\mathrm{A}^{\mathrm{III}} \mathrm{B}^{\mathrm{V}}$ слоев с сформированной податливой кремниевой подложкой, а также эффективно решить задачи, связанные с контролем деформации и остаточных напряжений в эпитаксиальном слое, что в итоге откроет новые пути создания эффективных устройств кремниевой электроники.

\section{Финансирование работы}

Работа выполнена при финансовой поддержке Российского научного фонда (грант № 19-72-10007). П.В. Середин выполнил свою часть исследования (рамановские исследования) при поддержке Министерства науки и высшего образования Российской Федерации (грант № ФЗГУ-2020-0036) в рамках государственного задания высшим учебным заведениям.

\section{Благодарности}

Рентгеновский исследования выполнены с привлечением оборудования ресурсного центра „Рентгенодифракционные методы исследования“ Санкт-Петербургского государственного университета.

\section{Конфоликт интересов}

Авторы заявляют, что у них нет конфликта интересов. 


\section{Список литературы}

[1] A. Fontcuberta i Morral. Nature, 580, 188 (2020). doi:10.1038/d41586-020-00976-8

[2] H. Kim, W.-J. Lee, A.C. Farrell, J.S.D. Morales, P. Senanayake, S.V. Prikhodko, T.J. Ochalski, D.L. Huffaker. Nano Lett., 17, 3465 (2017). doi:10.1021/acs.nanolett.7b00384

[3] Y.A. Bioud, A. Boucherif, M. Myronov, A. Soltani, G. Patriarche, N. Braidy, M. Jellite, D. Drouin, R. Arés. Nature Commun., 10, 4322 (2019). doi:10.1038/s41467-019-12353-9

[4] G. Gommé, G. Gautier, M. Portail, E. Frayssinet, D. Alquier, Y. Cordier, F. Semond. Phys. Status Solidi A, 214, 1600450 (2017). doi:10.1002/pssa.201600450

[5] S.H. Abud, A.M. Selman, Z. Hassan. Superlatt. Microstruct., 97, 586 (2016). doi:10.1016/j.spmi.2016.07.017

[6] P.V. Seredin, A.S. Lenshin, A.M. Mizerov, H. Leiste, M. Rinke. Appl. Surf. Sci., 476, 1049 (2019). doi:10.1016/j.apsusc.2019.01.239

[7] P.V. Seredin, H. Leiste, A.S. Lenshin, A.M. Mizerov. Appl. Surf. Sci., 508, 145267 (2020). doi:10.1016/j.apsusc.2020.145267

[8] M.R. Jimenéz-Vivanco, G. García, J. Carrillo, V. Agarwal, T. Díaz-Becerril, R. Doti, J. Faubert, J.E. Lugo. Sci. Rep., 10, 2220 (2020). doi:10.1038/s41598-020-59001-7

[9] W. Liu, X. Xie, M. Zhang, Q. Shen, C. Lin, L. Wang, P.K. Chu. J. Vac. Sci. Technol. B Microelectron. Nanometer Struct., 21, 168 (2003). doi:10.1116/1.1537714

[10] C.-C. Chiang, B.T.-H. Lee. Sci. Rep., 9, 12631 (2019). doi:10.1038/s41598-019-49119-8

[11] V.V. Starkov, E.A. Gosteva, D.M. Sedlovets, M.O. Kah. J. Electrochem. Soc., 165, E534 (2018). doi:10.1149/2.1101811jes

[12] A.S. Lenshin, P.V. Seredin, B.L. Agapov, D.A. Minakov, V.M. Kashkarov. Mater. Sci. Semicond. Process., 30, 25 (2015). doi:10.1016/j.mssp.2014.09.040

[13] A.S. Lenshin, V.M. Kashkarov, P.V. Seredin, D.A. Minakov, B.L. Agapov, M.A. Kuznetsova, V.A. Moshnikov, E.P. Domashevskaya. Semiconductors, 46, 1079 (2012). doi:10.1134/S1063782612080131

[14] J.L. Kulikowski, M. Przytulska, A. Chwojnowski. Biomed. Eng. OnLine, 17, 68 (2018). doi:10.1186/s12938-018-0481-9

[15] P. Elia, E.'Nativ-Roth, Y. Zeiri, Z. Porat. Microporous Mesoporous Mater., 225, 465 (2016). doi:10.1016/j.micromeso.2016.01.007

[16] Y.-I. Kang, W. Qiu, Z. Lei. Optoelectron. Lett., 3, 126 (2007). doi:10.1007/s11801-007-7022-2

[17] Z. Xu, Z. He, Y. Song, X. Fu, M. Rommel, X. Luo, A. Hartmaier, J. Zhang, F. Fang. Micromachines, 9, 361 (2018). doi:10.3390/mi9070361

[18] S. Manotas, F. Agulló-Rueda, J.D. Moreno, F. Ben-Hander, J.M. Martínez-Duart. Thin Sol. Films, 401, 306 (2001). doi:10.1016/S0040-6090(01)01641-8

[19] P.V. Seredin, A.S. Lenshin, D.S. Zolotukhin, I.N. Arsentyev, A.V. Zhabotinskiy, D.N. Nikolaev. Phys. E Low-Dim. Syst. Nanostruct., 97, 218 (2018). doi:10.1016/j.physe.2017.11.018

[20] Tauc J. Progr. Semicond. Heywood Lond., 9, 87 (1965).

\section{Properties of Compliant Porous Silicon-Based Substrates Formed by Two-Stage Etching}

\author{
P.V. Seredin ${ }^{1}$, A.S. Lenshin ${ }^{1}$, Abduljabbar Riyad \\ Khuder $^{2}$, D.L. Goloshchapov ${ }^{1}$, M.A. Kharajidi ${ }^{1}$, \\ I.N. Arsentiev ${ }^{3}$, I.A. Kasatkin ${ }^{4}$
}

${ }^{1}$ Voronezh State University, 394018 Voronezh, Russia

${ }^{2}$ Ministry of Education/General Directorate of Education in Baghdad,

The third Karkh Governorate, Iraq

${ }^{3}$ loffe Institute, 194021 St. Petersburg, Russia

${ }^{4}$ St. Petersburg State University, 199034 St. Petersburg, Russia

\begin{abstract}
We report on the effect of etching modes and their combination on the design, microstructural and optical properties of pliable substrates based on porous silicon. Based on the data of a complex of microstructural and spectroscopic methods of analysis, it was shown that, with constant parameters of the crystal lattice, the value of residual stresses, crystallite size, volume of the crystalline fraction, as well as the reflectivity and energy of direct transitions in the porous silicon layer depend on the combination of etching modes, but not always correlate with the value of the layer porosity calculated from the analysis of SEM images.
\end{abstract}

Редактор Г.А. Оганесян 